\title{
Phase spaces of Doubly Special Relativity
}

\author{
A. Błaut, M. Daszkiewicz \\ J. Kowalski-Glikman ${ }^{\ddagger}$, and S. Nowak ${ }^{\S}$ \\ Institute for Theoretical Physics \\ University of Wroctaw \\ Pl. Maxa Borna 9 \\ Pl-50-204 Wroctaw, Poland
}

October 23, 2018

\begin{abstract}
We show that depending on the direction of deformation of $\kappa$ Poincaré algebra (time-like, space-like, or light-like) the associated phase spaces of single particle in Doubly Special Relativity theories have the energy-momentum spaces of the form of de Sitter, anti-de Sitter, and flat space, respectively.
\end{abstract}

*email: ablaut@ift.uni.wroc.pl

†email: marcin@ift.uni.wroc.pl

$\ddagger$ email: jurekk@ift.uni.wroc.pl; Research partially supported by the KBN grant 5PO3B05620.

§email: pantera@ift.uni.wroc.pl 
It has been claimed for some time that quantum groups could play significant role in quantizing gravity (see, for example, 1] and also [2, where the role of quantum symmetries in $2+1$ quantum gravity has been explicitly shown.) If this claim is correct in the case of $3+1$ quantum gravity, then one can expect that the symmetries of "quantum special relativity" defined as a flat space limit of quantum gravity should also be properly defined in terms of quantum groups.

Following this intuition quantum $\kappa$-Poincaré algebra [3], 4] has been proposed some time ago as a posible algebra of symmetries of flat space in Planckian regime. This algebra was incorporated later to the construction of Doubly Special Relativity (DSR) theories [5, 6], 7], 8], in which it plays a role of the symmetry algebra of one-particle states (another version of DSR theory has been proposed in 9, 10, but it can be also described in terms of a $\kappa$-Poincaré algebra [11.)

It happened to be more natural to introduce DSR as a theory of energy and momenta with deformed action of boosts. It turns out, however that one can make use of the co-product of $\kappa$-Poincaré algebra to construct position sector of DSR. In particular it has been shown in [12, [13, [14] that in DSR one particle dynamics takes place in the phase space, whose energymomentum manifold forms four dimensional de Sitter space, while the space of positions is the non-commutative $\kappa$-Minkowski space [4. The link between $\kappa$-Minkowski space and $\kappa$-Poincaré algebra, which was originally obtained by using duality of Hopf algebra ([4], [15]), has been recently re-derived in the paper [16] starting from dynamics on $\kappa$-Minkowski space.

In the analysis reported in these papers the starting point was a particular form of $\kappa$-Poincaré algebra, with deformation along time-like direction. It is well known however that there are other $\kappa$-Poincaré algebras [17, [18] in which the deformation can be directed along light-like or space-like directions. In this paper we will present construction of phase spaces in such a general case.

Let us start with reviewing the general form of $\kappa$-Poincaré algebra (in the case of arbitrary Minkowski metric $g^{\mu \nu}$ ), whose algebraic part is given by [17]

$$
\begin{aligned}
{\left[M^{\mu \nu}, M^{\alpha \beta}\right] } & =i\left(g^{\mu \beta} M^{\nu \alpha}-g^{\nu \beta} M^{\mu \alpha}+g^{\nu \alpha} M^{\mu \beta}-g^{\mu \alpha} M^{\nu \beta}\right) \\
{\left[P_{\mu}, P_{\nu}\right] } & =0, \quad\left[M^{i j}, P_{0}\right]=0
\end{aligned}
$$




$$
\begin{aligned}
{\left[M^{i j}, P_{k}\right]=} & i \kappa\left(\delta_{k}^{j} g^{0 i}-\delta_{k}^{i} g^{0 j}\right)\left(1-\mathrm{e}^{-P_{0} / \kappa}\right)+i\left(\delta_{k}^{j} g^{i s}-\delta_{k}^{i} g^{j s}\right) P_{s} \\
{\left[M^{i 0}, P_{0}\right]=} & i \kappa g^{i 0}\left(1-\mathrm{e}^{-P_{0} / \kappa}\right)+i g^{i k} P_{k} \\
{\left[M^{i 0}, P_{k}\right]=} & -i \frac{\kappa}{2} g^{00} \delta_{k}^{i}\left(1-\mathrm{e}^{-2 P_{0} / \kappa}\right)-i \delta_{k}^{i} g^{0 s} P_{s} \mathrm{e}^{-P_{0} / \kappa} \\
& +i g^{0 i} P_{k}\left(\mathrm{e}^{-P_{0} / \kappa}-1\right)+\frac{i}{2 \kappa} \delta_{k}^{i} g^{r s} P_{r} P_{s}-\frac{i}{\kappa} g^{i s} P_{s} P_{k}
\end{aligned}
$$

Here Greek indices run from 0 to $D$, where $D+1$ is the dimension of spacetime under consideration, while Latin from 1 to $D$. It is easy to see that the standard $\kappa$-Poincaré algebra of DSR in the bicrossproduct basis is a particular example of the general algebra (11) (5), corresponding to the metric $g_{\mu \nu}=$ $\operatorname{diag}(-1,1,1,1)$ (in four spacetime dimensions) so that the deformation is in the time-like direction. One can consider other cases, however, with $g_{\mu \nu}=$ $\operatorname{diag}(1,-1,1,1)$, and

$$
g_{\mu \nu}=\left(\begin{array}{llll}
0 & 1 & 0 & 0 \\
1 & 0 & 0 & 0 \\
0 & 0 & 1 & 0 \\
0 & 0 & 0 & 1
\end{array}\right)
$$

corresponding to space-like and light-like [17, 18] deformation, respectively. It should be stressed that analogous construction can be made in any dimension $D \geq 1$.

According to [17 the algebra (1)-(5) with the following co-product structure

$$
\begin{aligned}
\Delta\left(P_{0}\right) & =1 \otimes P_{0}+P_{0} \otimes 1 \\
\Delta\left(P_{k}\right) & =P_{k} \otimes \mathrm{e}^{-P_{0} / \kappa}+1 \otimes P_{k} \\
\Delta\left(M^{i j}\right) & =M^{i j} \otimes 1+1 \otimes M^{i j} \\
\Delta\left(M^{i 0}\right) & =1 \otimes M^{i 0}+M^{i 0} \otimes \mathrm{e}^{-P_{0} / \kappa}-\frac{1}{\kappa} M^{i j} \otimes P_{j}
\end{aligned}
$$

and appropriately given antipode defines Hopf algebra, which we call quantum $\kappa$-Poincaré algebra with arbitrary metric $g_{\mu \nu}$. To extend this structure to the whole of the phase space of a one-particle system one adds the dual quantum group with generators being Lorentz transformations $\Lambda^{\mu}{ }_{\nu}$ and translations of momenta, which can be interpreted as positions $X^{\mu}$. Their co-products are 17]

$$
\Delta\left(X^{\mu}\right)=\Lambda_{\nu}^{\mu} \otimes X^{\nu}+X^{\mu} \otimes 1
$$


and

$$
\Delta\left(\Lambda_{\nu}^{\mu}\right)=\Lambda_{\rho}^{\mu} \otimes \Lambda_{\nu}^{\rho}
$$

Next one defines the pairings between elements of the algebra and the group as follows

$$
\begin{aligned}
\left\langle P_{\mu}, X^{\nu}\right\rangle & =i \delta_{\mu}^{\nu} \\
\left\langle\Lambda^{\mu}{ }_{\nu}, M^{\alpha \beta}\right\rangle & =i\left(g^{\alpha \mu} \delta_{\nu}^{\beta}-g^{\beta \mu} \delta_{\nu}^{\alpha}\right) \\
\left\langle\Lambda^{\mu}{ }_{\nu}, 1\right\rangle & =\delta_{\nu}^{\mu}
\end{aligned}
$$

The phase space brackets can be found by employing Heisenberg double procedure [19], 20], 12]

$$
\begin{aligned}
{\left[X^{\mu}, P_{\nu}\right] } & =P_{\nu(1)}\left\langle X_{(1)}^{\mu}, P_{\nu(2)}\right\rangle X_{(2)}^{\mu}-P_{\nu} X^{\mu} \\
{\left[X^{\mu}, M_{\sigma}^{\rho}\right] } & =M_{(1)}^{\rho}\left\langle\left\langle X_{(1)}^{\mu}, M_{(2)}^{\rho}\right\rangle X_{(2)}^{\mu}-M_{\sigma}^{\rho} X^{\mu}\right.
\end{aligned}
$$

where we make use of the standard ("Sweedler") notation for co-product

$$
\Delta \mathcal{T}=\sum \mathcal{T}_{(1)} \otimes \mathcal{T}_{(2)}
$$

In this way one gets the following phase space commutator constituting the so called $\kappa$-Minkowski, non-commutative spacetime

$$
\begin{aligned}
{\left[X^{\mu}, X^{\nu}\right] } & =-\frac{i}{\kappa} X^{\mu} \delta_{0}^{\nu}+\frac{i}{\kappa} X^{\nu} \delta_{0}^{\mu} \\
{\left[X^{\mu}, M^{k 0}\right] } & =i\left(g^{k \mu} X^{0}-g^{0 \mu} X^{k}\right)-\frac{i}{\kappa}\left(\delta_{0}^{\mu} M^{k 0}+\delta_{l}^{\mu} M^{k l}\right) \\
{\left[X^{\mu}, M^{k l}\right] } & =i\left(g^{k \mu} X^{l}-g^{l \mu} X^{k}\right)
\end{aligned}
$$

and the commutators between positions $X$ and momenta

$$
\left[P_{\nu}, X^{\mu}\right]=-i \delta_{\nu}^{\mu}+\frac{i}{\kappa} \delta_{0}^{\mu} P_{\sigma}\left(\delta_{\nu}^{\sigma}-\delta_{\nu}^{0} \delta_{\nu}^{\sigma}\right)
$$

It is interesting to ask which algebra the generators $X^{\mu}$ and $M^{\mu \nu}$ form. Because of (2)-(5) and (14) this $(D+1)(D+2) / 2$-dimensional algebra is the algebra of symmetries of the $D+1$ dimensional energy-momentum space. Assuming that the symmetries act on the energy-momentum space transitively, and that the subalgebra of symmetries leaving invariant the point $P_{\mu}=0$ is the Lorentz subalgebra, we see that energy-momentum space is isomorphic 
to the quotient of the group generated by $X$ and $M$ algebra by its Lorentz subgroup. Therefore knowing the algebra of $X$ and $M$ we know the form of energy-momentum manifold.

To find the algebra in question let us make the identification

$$
\hat{M}^{\mu \nu}=M^{\mu \nu}, \quad \hat{M}^{(D+1) \mu}=-\hat{M}^{\mu(D+1)}=\kappa X^{\mu}
$$

One can easily check that the algebra of $(D+1)(D+2) / 2$ generators $\hat{M}^{A B}$, $(A, B=0, \ldots D+1)$ satisfying

$$
\left[\hat{M}^{A B}, \hat{M}^{C D}\right]=i\left(\hat{g}^{B C} \hat{M}^{A D}-\hat{g}^{A C} \hat{M}^{B D}-\hat{g}^{B D} \hat{M}^{A C}+\hat{g}^{A D} \hat{M}^{B C}\right)
$$

is equal to (11)-(13) if

$$
\hat{g}^{A B}=\left(\begin{array}{cccc} 
& & 1 \\
& & & 0 \\
& & & \vdots \\
& & 0 \\
1 & 0 \cdots 0 & 0
\end{array}\right)
$$

where

$$
g^{\mu \nu}=\left(\begin{array}{ccc}
g^{00} & \cdots & g^{0 D} \\
\vdots & \ddots & \vdots \\
g^{D 0} & \cdots & g^{D D}
\end{array}\right)
$$

is the Minkowski spacetime metric in the Hopf algebra (11)-(10).

First we ask if the metric $\hat{g}^{A B}$ can be degenerate, i.e., if it can have zero eigenvalues. Since the metric $g^{\mu \nu}$ is non-degenerate (with eigenvalues $-1,1, \ldots, 1)$ from standard theorems of matrix algebra it follows that the rank of $\hat{g}^{A B}$ can be at least $D+1$, and thus it can have at most one zero eigenvalue. This happens if, for example, one of the first $D+1$ columns of $\hat{g}^{A B}$ is proportional to its last column, i.e., in the light-like case. Straightforward algebraic considerations show next that $\hat{g}^{A B}$ can have only the following signatures: $[-,+, \ldots,+],[-,-,+, \ldots,+]$ or $[0,-,+, \ldots,+]$ corresponding to the time-like, space-like and light-like deformations, respectively.

Let us now make this explicit in the four-dimensional case.

Time-like deformation. In this case $g^{\mu \nu}=\operatorname{diag}(-1,1,1,1)$ and the algebra (15) is the $S O(1,4)$ algebra, while the energy-momentum space is de 
Sitter space $S O(1,4) / S O(1,3)$. This case has been analyzed before in [13] and [14.

Space-like deformation. $g^{\mu \nu}=\operatorname{diag}(1,-1,1,1)$ and the algebra (15) is the $S O(2,3)$ algebra. The energy-momentum space is anti-de Sitter space $S O(2,3) / S O(1,3)$. It is worth noticing that anti-de Sitter space is the energymomentum manifold of $2+1$ dimensional quantum gravity coupled to point particle [22]. Explicitly, the relation between the generators $J^{\mu}$ (rotation and boosts) and $y^{\mu}$ (translations) employed in that paper, which satisfy the algebra

$$
\begin{aligned}
{\left[y^{\mu}, y^{\nu}\right] } & =2 \epsilon^{\mu \nu}{ }_{\sigma} y^{\sigma} \\
{\left[J^{\mu}, J^{\nu}\right] } & =-\epsilon^{\mu \nu}{ }_{\sigma} J^{\sigma} \\
{\left[J^{\mu}, y^{\nu}\right] } & =-\epsilon^{\mu \nu}{ }_{\sigma} y^{\sigma}
\end{aligned}
$$

and our generators is the following

$$
\begin{aligned}
& J^{1}=-i \kappa X^{0} \\
& J^{2}=i M^{01} \\
& J^{0}=i \kappa X^{1}-i M^{01} \\
& y^{1}=i \kappa X^{0}+i M^{12} \\
& y^{2}=-i \kappa X^{2}-i M^{01}+i M^{02} \\
& y^{0}=-i \kappa X^{1}+i M^{01}-M^{02}
\end{aligned}
$$

The relation of $2+1$ quantum gravity to DSR theories and its possible relevance for $3+1$ dimensional physics has been analyzed in [23] and [24].

Light-like deformation. Now the metric $g^{\mu \nu}$ is given by (6) and it is clear that the metric $\hat{g}^{A B}$ is degenerate. In context of DSR theory such deformation has been analyzed in [21. It is easy to see that in this case the algebra (15) is the standard Poincare algebra and that the energy-momentum space is the flat Minkowski space. Explicitly, the four commuting elements $\mathcal{X}^{\mu}$ have the form

$$
\mathcal{X}^{0}=X^{0}-\frac{1}{\kappa} M^{10}, \quad \mathcal{X}^{1}=X^{1}, \quad \mathcal{X}^{\alpha}=X^{\alpha}-\frac{1}{\kappa} M^{1 \alpha}, \quad \alpha=2,3 .
$$

This case certainly deserves further studies, as it may correspond to DSR theories with commuting spacetime. There is a hope therefore that, for example, the construction of $\kappa$-deformed field theory [18] might be much simpler here 
than in other cases. We will present the results of this investigations in a separate paper.

In conclusions let us recapitulate the results of reported above. We found that in general case, there are three DSR phase spaces associated with $\kappa$ Poincaré algebra. Let us stress that since this algebra has the same form in any dimension, our results hold for any space dimension $D \geq 1$ as well. It is of course an open problem which of this cases (if any) is a correct setting for $3+1$ quantum special relativity. Work in this direction is in progress.

Acknowledgement. We would like to thank P. Ługiewicz and J. Lukierski for discussion and useful comments.

\section{References}

[1] S. Major and L. Smolin, "Quantum deformation of quantum gravity," Nucl. Phys. B 473 (1996) 267 arXiv:gr-qc/9512020.

[2] F. A. Bais, N. M. Muller and B. J. Schroers, "Quantum group symmetry and particle scattering in $(2+1)$-dimensional quantum gravity," Nucl. Phys. B 640 (2002) 3 arXiv:hep-th/0205021.

[3] J. Lukierski, H. Ruegg, A. Nowicki and V. N. Tolstoi, "Q deformation of Poincare algebra," Phys. Lett. B 264 (1991) 331.

[4] S. Majid and H. Ruegg, "Bicrossproduct structure of kappa Poincare group and noncommutative geometry," Phys. Lett. B 334 (1994) 348 arXiv:hep-th/9405107; J. Lukierski, H. Ruegg and W. J. Zakrzewski, "Classical and quantum mechanics of free kappa relativistic systems," Annals Phys. 243 (1995) 90 arXiv:hep-th/9312153.

[5] G. Amelino-Camelia, "Testable scenario for relativity with minimumlength," Phys. Lett. B 510 (2001) 255 arXiv:hep-th/0012238.

[6] G. Amelino-Camelia, "Relativity in space-times with short-distance structure governed by an observer-independent (Planckian) length scale," Int. J. Mod. Phys. D 11 (2002) 35 arXiv:gr-qc/0012051.

[7] J. Kowalski-Glikman, "Observer independent quantum of mass," Phys. Lett. A 286 (2001) 391 arXiv:hep-th/0102098. 
[8] N. R. Bruno, G. Amelino-Camelia and J. Kowalski-Glikman, "Deformed boost transformations that saturate at the Planck scale," Phys. Lett. B 522 (2001) 133 arXiv:hep-th/0107039.

[9] J. Magueijo and L. Smolin, "Lorentz invariance with an invariant energy scale," Phys. Rev. Lett. 88 (2002) 190403 arXiv:hep-th/0112090.

[10] J. Magueijo and L. Smolin, "Generalized Lorentz invariance with an invariant energy scale," Phys. Rev. D 67 (2003) 044017 arXiv:gr-qc/0207085.

[11] J. Kowalski-Glikman and S. Nowak, "Doubly special relativity theories as different bases of kappa-Poincare algebra," Phys. Lett. B 539 (2002) 126 arXiv:hep-th/0203040.

[12] J. Kowalski-Glikman and S. Nowak, "Non-commutative space-time of doubly special relativity theories," Int. J. Mod. Phys. D 12 (2003) 299 arXiv:hep-th/0204245.

[13] J. Kowalski-Glikman, "De Sitter space as an arena for doubly special relativity," Phys. Lett. B 547 (2002) 291 arXiv:hep-th/0207279.

[14] J. Kowalski-Glikman and S. Nowak, "Doubly special relativity and de Sitter space," Class. Quant. Grav. 20 (2003) 4799 arXiv:hep-th/0304101.

[15] S. Zakrzewski, J. Phys. A 27, 2075 (1994).

[16] A. Agostini, G. Amelino-Camelia and F. D'Andrea, "Hopfalgebra description of noncommutative-spacetime symmetries," arXiv:hep-th/0306013.

[17] P. Kosinski and P. Maslanka, "The $\kappa$-Weyl group and its algebra", arXiv: q-alg/9512018; in From Field Theory to Quantum Groups, ed. B. Jancewicz, J. Sobczyk, p. 41, World Scientific (1996).

[18] P. Kosinski, P. Maslanka, J. Lukierski and A. Sitarz, "Generalized kappa-deformations and deformed relativistic scalar fields on noncommutative Minkowski space," arXiv:hep-th/0307038.

[19] A. Nowicki, "Kappa-Deformed Phase Space and Uncertainty Relations," arXiv:math.QA/9803064. 
[20] J. Lukierski and A. Nowicki, Proceedings of Quantum Group Symposium at Group 21, (July 1996, Goslar) Eds. H.-D. Doebner and V.K. Dobrev, Heron Press, Sofia, 1997, p. 186.

[21] A. Błaut, M. Daszkiewicz and J. Kowalski-Glikman, "Doubly special relativity with light-cone deformation," Mod. Phys. Lett. A 18 (2003) 1711 arXiv:hep-th/0302157.

[22] H. J. Matschull and M. Welling, "Quantum mechanics of a point particle in 2+1 dimensional gravity," Class. Quant. Grav. 15 (1998) 2981 arXiv:gr-qc/9708054.

[23] G. Amelino-Camelia, L. Smolin and A. Starodubtsev, "Quantum symmetry, the cosmological constant and Planck scale phenomenology," arXiv:hep-th/0306134

[24] L. Freidel, J. Kowalski-Glikman and L. Smolin, " $2+1$ gravity and doubly special relativity," arXiv:hep-th/0307085. 